\title{
On Hadamard Product of P-Valent Functions with Alternating
} Type

\author{
${ }^{1}$ S.S.Pokley, ${ }^{2}$ K.D.Patil, ${ }^{3}$ M.G.Shrigan \\ ${ }^{I}$ Department of Mathematics, Kavikulguru Institute of Technology and Science, Ramtek-441106, (M.S.), India \\ ${ }^{2}$ Department of Mathematics, Bapurao Deshmukh College of Engineering, Sevagram Wardha-442102(M.S.), \\ India \\ ${ }^{3}$ Department of Mathematics, G.H.Raisoni Institute of Engineering \& Technology, Pune - 412207, (M.S.), India
}

Abstract: Padmanabhan and Ganeshan have obtained some results on Hadamard product of univalent functions with negative coefficients of the type $\mathrm{f}(\mathrm{z})=\mathrm{z}-\sum_{\mathrm{n}=1}^{\infty} \mathrm{a}_{2 \mathrm{n}} \mathrm{z}^{2 \mathrm{n}}, \mathrm{a}_{2 \mathrm{n}} \geq 0$. In this paper we have obtained coefficient bounds and convolution results of p-valent function.

Keywords: Hadamard (convolution) product, -Valent function, Cauchy-Schwarz inequality.

Mathematics Subject Classification (2000): 30C45, 30C50

\section{Introduction}

Let $\mathcal{A}$ (p) denote the class of $f$ normalized univalent functions of the form

$$
f(z)=z^{p}+\sum_{n=p}^{\infty}(-1)^{n+1} a_{n+1} z^{n+1} \quad, a_{n+1} \geq 0
$$

holomorphic \& p-valent in the unit disc $\mathrm{E}=\{\mathrm{z}: \mathrm{z} \in \mathrm{C} ;|\mathrm{z}|<1\}$.

A function $\mathrm{f} \in \mathcal{A}(\mathrm{p})$ is said to belong to the class $\mathrm{S}(\mathrm{p}, \alpha)$ of p-valently starlike function of order $\alpha(0 \leq$ $\alpha \leq p)$ if it satisfies, for $z \in E$, the condition

$$
\operatorname{Re}\left(\frac{z f^{\prime}(z)}{f(z)}\right)>\alpha
$$

A function $\mathrm{f} \in \mathcal{A}(\mathrm{p})$ is said to belong to the class $\mathrm{S}(\mathrm{p}, \alpha)$ of $\mathrm{p}$-valently convex functions of order $\alpha(0 \leq \alpha \leq \mathrm{p})$ if it satisfies, for $\mathrm{z} \in \mathrm{E}$, the condition

$$
\operatorname{Re}\left(1+\frac{z f^{\prime \prime}(z)}{f^{\prime}(z)}\right)>\alpha
$$

The class $\mathrm{S}(\mathrm{p}, \alpha)$ was introduced by Goodman [1].

Silverman [2] studied the properties of these functions. Let $K=\{w / w$ is analytic in $E ; w(0)=0,|w(z)|$

$<1$ in $\mathrm{E}$ \}. Let $\mathrm{G}(\mathrm{A}, \mathrm{B})$ denote a subclass of analytic functions in $\mathrm{E}$, which are of the form $\frac{1+\mathrm{Aw}(\mathrm{z})}{1+\mathrm{Bw}(\mathrm{z})},-1 \leq$

$\mathrm{A}<\mathrm{B} \leq 1$ where $\mathrm{w}(\mathrm{z}) \in \mathrm{K}$.

Consider the following definitions,

$$
\begin{aligned}
& \mathrm{S}^{*}(A, B)=\left\{f / f \in \mathrm{S} \text { and } \frac{z f^{\prime}}{f} \in G(A, B)\right\} \\
& H(A, B)=\left\{f / f \in \mathrm{S} \text { and }\left(\frac{z f}{f^{\prime}}\right) \in G(A, B)\right\} \\
& \mathrm{M}^{*}(A, B)=\left\{f / f \in M \text { and } \frac{z f}{f} \in G(A, B)\right\}
\end{aligned}
$$




$$
C(A, B)=\left\{f / f \in S \text { and }\left(\frac{z f^{\prime}}{f^{\prime}}\right)^{\prime} \in G(A, B)\right\}
$$

If $\quad \mathrm{f}(\mathrm{z})=\mathrm{z}^{\mathrm{p}}+\sum_{\mathrm{n}=\mathrm{p}}^{\infty}(-1)^{\mathrm{n}+1} \mathrm{a}_{\mathrm{n}+1} \mathrm{z}^{\mathrm{n}+1} \quad, \mathrm{a}_{\mathrm{n}+1} \geq 0$

and

$$
\mathrm{g}(\mathrm{z})=\mathrm{z}^{\mathrm{p}}+\sum_{\mathrm{n}=\mathrm{p}}^{\infty}(-1)^{\mathrm{n}+1} \mathrm{~b}_{\mathrm{n}+1} \mathrm{z}^{\mathrm{n}+1} \quad, \mathrm{~b}_{\mathrm{n}+1} \geq 0
$$

The class $\mathcal{A}$ (p) is closed under the Hardmard product (or Convolution) of $\mathrm{f} \& \mathrm{~g}$ as,

$$
\mathrm{h}(\mathrm{z})=\mathrm{f}(\mathrm{z}) * \mathrm{~g}(\mathrm{z})=\mathrm{z}^{\mathrm{p}}+\sum_{\mathrm{n}=\mathrm{p}}^{\infty}(-1)^{\mathrm{n}+1} \mathrm{a}_{\mathrm{n}+1} \mathrm{~b}_{\mathrm{n}+1} \mathrm{z}^{\mathrm{n}+1} \quad, \mathrm{a}_{\mathrm{n}+1}, \mathrm{~b}_{\mathrm{n}+1} \geq 0
$$

K..S. Padmanabhan and M.S. Ganeshan [3] also S.M.Khainar and Meena More [4] studied some convolution properties of functions with negative coefficients. In this paper we extend the results on convolution property for p-valent function in the class $\mathrm{M}^{*}(\mathrm{~A}, \mathrm{~B})$ and $\mathrm{C}(\mathrm{A}, \mathrm{B})$.

\section{Preliminary and Main Results}

We start with lemma which will be required for further investigation convolution results.

Leema 2.1: A function

$$
\mathrm{f}(\mathrm{z})=z^{\mathrm{p}}+\sum_{\mathrm{n}=\mathrm{p}}^{\infty}(-1)^{\mathrm{n}+1} \mathrm{a}_{\mathrm{n}+1} \mathrm{z}^{\mathrm{n}+1} \quad, \mathrm{a}_{\mathrm{n}+1} \geq 0
$$

is in $M^{*}(A, B)$ iff,

$$
\left(\frac{(p-1)+\sum_{n=p}^{\infty}[n(B+1)-(A-B)] a_{n+1}}{(A-p B)}\right) \leq 1 .
$$

Proof : We have

$$
\mathrm{f}(\mathrm{z})=z^{\mathrm{p}}+\sum_{\mathrm{n}=\mathrm{p}}^{\infty}(-1)^{\mathrm{n}+1} \mathrm{a}_{\mathrm{n}+1} \mathrm{z}^{\mathrm{n}+1} \quad, \mathrm{a}_{\mathrm{n}+1} \geq 0
$$

then

$$
\frac{\mathrm{z} f^{\prime}(z)}{f(z)}=\frac{\mathrm{p} z^{\mathrm{p}}+\sum_{\mathrm{n}=\mathrm{p}}^{\infty}(-1)^{\mathrm{n}+1} \mathrm{a}_{\mathrm{n}+1}(\mathrm{n}+1) \mathrm{z}^{\mathrm{n}+1}}{z^{\mathrm{p}}+\sum_{\mathrm{n}=\mathrm{p}}^{\infty}(-1)^{\mathrm{n}+1} \mathrm{a}_{\mathrm{n}+1} \mathrm{z}^{\mathrm{n}+1}} \in G(A, B)
$$

iff

$$
\frac{p z^{p}+\sum_{n=p}^{\infty}(-1)^{n+1} a_{n+1}(n+1) z^{n+1}}{z^{p}+\sum_{n=p}^{\infty}(-1)^{n+1} a_{n+1} z^{n+1}}=\frac{1+A w(z)}{1+B w(z)}
$$


On Simplification, we get

$\mathrm{w}(\mathrm{z})\left\{(\mathrm{A}-\mathrm{pB}) \mathrm{z}^{\mathrm{p}}+\sum_{\mathrm{n}=\mathrm{p}}^{\infty}[\mathrm{A}-\mathrm{B}(\mathrm{n}+1)](-1)^{\mathrm{n}+1} \mathrm{a}_{\mathrm{n}+1} \mathrm{z}^{\mathrm{n}+1}\right\}=(\mathrm{p}-1) \mathrm{z}^{\mathrm{p}}+\sum_{\mathrm{n}=\mathrm{p}}^{\infty} \mathrm{n}(-1)^{\mathrm{n}+1} \mathrm{a}_{\mathrm{n}+1} \mathrm{z}^{\mathrm{n}+1}$

Therefore

$$
w(z)=\frac{(p-1) z^{p}+\sum_{n=p}^{\infty} n(-1)^{n+1} a_{n+1} z^{n+1}}{\left\{(A-p B) z^{p}+\sum_{n=p}^{\infty}[A-B(n+1)](-1)^{n+1} a_{n+1} z^{n+1}\right\}}
$$

Notice that $|\mathrm{w}(\mathrm{z})| \leq 1$, we have

Allowing $|\mathrm{z}|=\mathrm{r} \rightarrow 1$,we get

$$
\left|\frac{(p-1) z^{p}+\sum_{n=p}^{\infty} n(-1)^{n+1} a_{n+1} z^{n+1}}{\left\{(A-p B) z^{p}+\sum_{n=p}^{\infty}[A-B(n+1)](-1)^{n+1} a_{n+1} z^{n+1}\right\}}\right| \leq 1
$$

Therefore

$$
\frac{(p-1)+\sum_{n=p}^{\infty} n a_{n+1}}{(A-p B)+\sum_{\mathrm{n}=\mathrm{p}}^{\infty}[\mathrm{A}-\mathrm{B}(\mathrm{n}+1)] \mathrm{a}_{\mathrm{n}+1}} \leq 1
$$

$$
\begin{aligned}
& (p-1)+\sum_{\mathrm{n}=\mathrm{p}}^{\infty}[\mathrm{n}(\mathrm{B}+1)-(\mathrm{A}-\mathrm{B})] \mathrm{a}_{\mathrm{n}+1} \leq A-p B \\
& \left(\frac{(p-1)+\sum_{n=p}^{\infty}[n(B+1)-(A-B)] a_{n+1}}{(A-p B)}\right) \leq 1
\end{aligned}
$$

Leema 2.2: A function

$$
f(z)=z^{p}+\sum_{n=p}^{\infty}(-1)^{n+1} a_{n+1} z^{n+1} \quad, a_{n+1} \geq 0
$$

is inc $(A, B)$ iff,

$$
\left(\frac{\left(p^{2}-p\right)+\sum_{n=p}^{\infty}(n+1)[n(B+1)-(A-B)] a_{n+1}}{p(A-p B)}\right) \leq 1
$$

Proof : We have

$$
\mathrm{f}(\mathrm{z})=z^{\mathrm{p}}+\sum_{\mathrm{n}=\mathrm{p}}^{\infty}(-1)^{\mathrm{n}+1} \mathrm{a}_{\mathrm{n}+1} \mathrm{z}^{\mathrm{n}+1} \quad, \mathrm{a}_{\mathrm{n}+1} \geq 0
$$


then

$$
\frac{\left(\mathrm{zf}^{\prime}(\mathrm{z})\right)^{\prime}}{\mathrm{f}^{\prime}(\mathrm{z})}=\frac{\mathrm{p}^{2} \mathrm{z}^{\mathrm{p}-1}+\sum_{\mathrm{n}=\mathrm{p}}^{\infty}(-1)^{\mathrm{n}+1} \mathrm{a}_{\mathrm{n}+1}(\mathrm{n}+1)^{2} \mathrm{z}^{\mathrm{n}}}{\mathrm{pz^{ \textrm {p } - 1 }}+\sum_{\mathrm{n}=\mathrm{p}}^{\infty}(-1)^{\mathrm{n}+1} \mathrm{a}_{\mathrm{n}+1}(\mathrm{n}+1) \mathrm{z}^{\mathrm{n}}} \in \mathrm{G}(\mathrm{A}, \mathrm{B})
$$

iff

$$
\begin{gathered}
\frac{\mathrm{p}^{2} \mathrm{z}^{\mathrm{p}-1}+\sum_{\mathrm{n}=\mathrm{p}}^{\infty}(-1)^{\mathrm{n}+1} \mathrm{a}_{\mathrm{n}+1}(\mathrm{n}+1)^{2} \mathrm{z}^{\mathrm{n}}}{\mathrm{p} \mathrm{z}^{\mathrm{p}-1}+\sum_{\mathrm{n}=\mathrm{p}}^{\infty}(-1)^{\mathrm{n}+1} \mathrm{a}_{\mathrm{n}+1}(\mathrm{n}+1) \mathrm{z}^{\mathrm{n}}}=\frac{1+\mathrm{Aw}(\mathrm{z})}{1+\mathrm{Bw}(\mathrm{z})} \\
\mathrm{w}(\mathrm{z})\left\{\left(\mathrm{PA}-\mathrm{P}^{2} \mathrm{~B}\right) \mathrm{z}^{\mathrm{p}-1}+\sum_{\mathrm{n}=\mathrm{p}}^{\infty}(-1)^{\mathrm{n}+1}\left[\mathrm{~A}(\mathrm{n}+1)-\mathrm{B}(\mathrm{n}+1)^{2}\right] \mathrm{a}_{\mathrm{n}+1} \mathrm{z}^{\mathrm{n}}\right\} \\
=\left(\mathrm{p}^{2}-\mathrm{p}\right) \mathrm{z}^{\mathrm{p}-1}+\sum_{\mathrm{n}=\mathrm{p}}^{\infty}(-1)^{\mathrm{n}+1}[\mathrm{n}(\mathrm{n}+1)] \mathrm{a}_{\mathrm{n}+1} \mathrm{z}^{\mathrm{n}}
\end{gathered}
$$

Again since $|w(z)| \leq 1$, we get

$$
\left|\frac{\left(p^{2}-p\right) z^{p-1}+\sum_{n=p}^{\infty}(-1)^{n+1}[n(n+1)] a_{n+1} z^{n}}{\left(P A-P^{2} B\right) z^{p-1}+\sum_{n=p}^{\infty}(-1)^{n+1}\left[A(n+1)-B(n+1)^{2}\right] a_{n+1} z^{n}}\right| \leq 1
$$

Allowing $|z|=r \rightarrow 1$ we get

$$
\begin{aligned}
& \left|\frac{\mathrm{p}(\mathrm{p}-1)+\sum_{\mathrm{n}=\mathrm{p}}^{\infty}[\mathrm{n}(\mathrm{n}+1)] \mathrm{a}_{\mathrm{n}+1} \mathrm{z}^{\mathrm{n}}}{\mathrm{p}(\mathrm{A}-\mathrm{PB})+\sum_{\mathrm{n}=\mathrm{p}}^{\infty}\left[\mathrm{A}(\mathrm{n}+1)-\mathrm{B}(\mathrm{n}+1)^{2}\right] \mathrm{a} n+1}\right| \leq 1 \\
& \left|\frac{\mathrm{p}(\mathrm{p}-1)+\sum_{\mathrm{n}=\mathrm{p}}^{\infty}[\mathrm{n}(\mathrm{n}+1)] \mathrm{a}_{\mathrm{n}+1} \mathrm{z}^{\mathrm{n}}}{\mathrm{p}(\mathrm{A}-\mathrm{PB})+\sum_{\mathrm{n}=\mathrm{p}}^{\infty}\left[\mathrm{A}(\mathrm{n}+1)-\mathrm{B}(\mathrm{n}+1)^{2}\right] \mathrm{a}_{\mathrm{n}+1}}\right| \leq 1 \\
& \frac{\left(p^{2}-p\right)+\sum_{n=p}^{\infty}(n+1)[(B+1) n-(A-B)] a_{n+1} \leq p(A-P B)}{\left(p^{2}-p\right)+\sum_{n=p}^{\infty}(n+1)[(B+1) n-(A-B)] a_{n+1}} \leq 1 \\
& p(A-P B)
\end{aligned}
$$

We define

$$
h(z)=f(z) * g(z)=z^{p}+\sum_{n=p}^{\infty}(-1)^{n} a_{n+1} b_{n+1} z^{n+1} \quad, a_{n+1}, b_{n+1} \geq 0
$$

for $f(z)$ and $g(z)$ members of $M^{*}(A, B)$ and $C(A, B)$. 
Theroem 2.1: A function

$$
\begin{array}{cc}
f(z)=z^{p}+\sum_{n=p}^{\infty}(-1)^{n+1} a_{n+1} z^{n+1} & , a_{n+1} \geq 0 \\
g(z)=z^{p}+\sum_{n=p}^{\infty}(-1)^{n+1} b_{n+1} z^{n+1} & , b_{n+1} \geq 0
\end{array}
$$

are elements of class $M^{*}(A, B)$ then

$$
h(z)=f(z) * g(z)=z^{p}+\sum_{n=p}^{\infty}(-1)^{n} a_{n+1} b_{n+1} z^{n+1}, a_{n+1}, b_{n+1} \geq 0
$$

is element of $M^{*}(A, B)$ with $-1 \leq A_{1} \leq B_{1} \leq 1$, where $A_{1} \geq-1, B_{1} \leq \frac{A_{1}-2 K}{1+2 K}$ these bounds for $A_{1}$ and $B_{1}$ are sharp.

Proof : Bylemma1, We have

$$
\left(\frac{(p-1)+\sum_{n=p}^{\infty}[n(B+1)-(A-B)] a_{n+1}}{(A-p B)}\right) \leq 1
$$

Since $f \in \mathrm{M}^{*}(\mathrm{~A}, \mathrm{~B})$.

$$
\left(\frac{(p-1)+\sum_{n=p}^{\infty}[n(B+1)-(A-B)] b_{n+1}}{(A-p B)}\right) \leq 1
$$

Since $g \in \mathrm{M}^{*}(\mathrm{~A}, \mathrm{~B})$.

We want to find $A_{1} \& B_{1}$ such that $-1 \leq A_{1} \leq B_{1} \leq 1$, for $h(z)=f(z) * g(z) \in \mathrm{M}^{*}(\mathrm{~A}, \mathrm{~B})$.

Now $h(z) \in \mathrm{M}^{*}(\mathrm{~A}, \mathrm{~B})$ if 


$$
\begin{gathered}
\left(\frac{(p-1)+\sum_{n=p}^{\infty}\left[n\left(B_{1}+1\right)-\left(A_{1}-B_{1}\right)\right] a_{n+1} b_{n+1}}{\left(A_{1}-p B_{1}\right)}\right) \leq 1 ; \text { by lemma } 2.1 \\
\left(\frac{(p-1)}{\left(A_{1}-p B_{1}\right)}+\sum_{n=p}^{\infty} \frac{\left[n\left(B_{1}+1\right)-\left(A_{1}-B_{1}\right)\right] a_{n+1} b_{n+1}}{\left(A_{1}-p B_{1}\right)}\right) \leq 1 \\
\frac{(p-1)}{\left(A_{1}-p B_{1}\right)}+\sum_{n=p}^{\infty} u_{1} a_{n+1} b_{n+1} \leq 1 \\
\text { where } u_{1}=\frac{\left[n\left(B_{1}+1\right)-\left(A_{1}-B_{1}\right)\right]}{\left(A_{1}-p B_{1}\right)}
\end{gathered}
$$

By Cauchy Schwarz inequlity,

$$
\begin{array}{r}
\sum_{n=p}^{\infty} \sqrt{u a_{n+1} b_{n+1}} \leq\left(\sum_{n=p}^{\infty} u a_{n+1}\right)^{\frac{1}{2}}\left(\sum_{n=p}^{\infty} u b_{n+1}\right)^{\frac{1}{2}} \leq 1 \\
\text { where } u=\frac{[n(B+1)-(A-B)]}{(A-p B)}
\end{array}
$$

(1.3) is true if

$$
\frac{(p-1)}{\left(A_{1}-p B_{1}\right)}+u_{1} a_{n+1} b_{n+1} \leq \frac{(p-1)}{(A-p B)}+u \sqrt{a_{n+1} b_{n+1}}
$$

If $A_{1}=A$ and $B_{1}=B$ then we get,

$$
u_{1} a_{n+1} b_{n+1} \leq u \sqrt{a_{n+1} b_{n+1}}
$$

i.e.

$$
u_{1} \sqrt{a_{n+1} b_{n+1}} \leq u
$$

Therefore it is enough to find $u$ such that

On Simplification, we get

$$
\frac{1}{u} \leq \frac{u}{u_{1}} \quad \text { i.e. } \quad u_{1} \leq u^{2}
$$




$$
\begin{aligned}
& \frac{\left[(p-1)+n\left(B_{1}+1\right)-\left(A_{1}-B_{1}\right)\right]}{\left(A_{1}-p B_{1}\right)} \leq\left\{\frac{[(p-1)+n(B+1)-(A-B)]}{(A-p B)}\right\}^{2}=u^{2} \\
& \frac{\left[(p-1)+n\left(B_{1}+1\right)-\left(A_{1}-B_{1}\right)\right]}{\left(A_{1}-p B_{1}\right)} \leq u^{2}
\end{aligned}
$$

i.e. $(p-1)+n\left(B_{1}+1\right)-\left(A_{1}-B_{1}\right) \leq\left(A_{1}-p B_{1}\right) u^{2}$

$$
A_{1} \geq \frac{(p-1)+\left(B_{1}+1\right) n+B_{1}\left(p u^{2}+1\right)}{\left(1+u^{2}\right)}
$$

Taking $B_{1}=1$ and $n=p$ in (1.8) and (1.5) above we get

$$
\begin{aligned}
A_{1} & \geq \frac{(p-1)+2 p+\left(p u^{2}+1\right)}{\left(1+u^{2}\right)} \\
A_{1} & \geq \frac{p\left(3+u^{2}\right)}{\left(u^{2}+1\right)} \\
& \geq p\left[1+2 \frac{(A-p B)^{2}}{(A-p B)^{2}+\left[(p-1)+p(B+1)-(A-B)^{2}\right]}\right] \\
A_{1} & \geq p(1+2 k)
\end{aligned}
$$

where $k=\frac{(A-p B)^{2}}{(A-p B)^{2}+\left[(p-1)+p(B+1)-(A-B)^{2}\right]}$

Theroem 2.2: If $f(z) \in M^{*}(A, B)$ and $g(z) \in M^{*}\left(A^{\prime}, B^{\prime}\right)$ then $f(z) * g(z) \in M^{*}\left(A_{1}, B_{1}\right)$

where $A_{1} \geq 1, B_{1} \leq \frac{A_{1}-2 k}{1+2 k}$ with

$$
k=\frac{(A-p B)\left(A^{\prime}-p B^{\prime}\right)}{\left(3 B_{1}+p-A_{1}+1\right)\left(3 B^{\prime}+p-A^{\prime}+1\right)+\left[\left(A_{1}-B_{1}\right)-(P-1)\right]\left(A^{\prime}-P B^{\prime}\right)}
$$

Proof :Proceeding with the arg ument developed in Theroem 1, we require

$$
\frac{\left[(p-1)+n\left(B_{1}+1\right)-\left(A_{1}-B_{1}\right)\right]}{\left(A_{1}-p B_{1}\right)} \leq \frac{\left[(p-1)+n\left(B_{1}+1\right)-\left(A_{1}-B_{1}\right)\right]}{\left(A_{1}-p B_{1}\right)} \frac{\left[(p-1)+n\left(B^{\prime}+1\right)-\left(A^{\prime}-B^{\prime}\right)\right]}{\left(A^{\prime}-p B^{\prime}\right)}=\alpha
$$

That is

$$
\begin{aligned}
& \frac{(p-1)+n\left(B_{1}+1\right)}{\left(A_{1}-p B_{1}\right)} \leq \alpha+\frac{A_{1}-B_{1}}{A_{1}-p B_{1}} \\
& \frac{A_{1}-p B_{1}}{B_{1}+1} \geq \frac{n\left(A_{1}-p B_{1}\right)}{\alpha\left(A_{1}-p B_{1}\right)+\left(A_{1}-B_{1}\right)-(p-1)}
\end{aligned}
$$

Notice that $\frac{n\left(A_{1}-p B_{1}\right)}{\alpha\left(A_{1}-p B_{1}\right)+\left(A_{1}-B_{1}\right)-(p-1)}$ decreases as $n$ increases.Simplifying, we get 


$$
\frac{A_{1}-p B_{1}}{B_{1}+1} \geq \frac{n(A-p B)\left(A^{\prime}-p B^{\prime}\right)}{\left(3 B_{1}+p-A_{1}+1\right)\left(3 B^{\prime}+p-A^{\prime}+1\right)+\left[\left(A_{1}-B_{1}\right)-(P-1)\right]\left(A^{\prime}-P B^{\prime}\right)}
$$

Taking $n=2$, we get

$$
\frac{A_{1}-p B_{1}}{B_{1}+1} \geq \frac{2(A-p B)\left(A^{\prime}-p B^{\prime}\right)}{\left(3 B_{1}+p-A_{1}+1\right)\left(3 B^{\prime}+p-A^{\prime}+1\right)+\left[\left(A_{1}-B_{1}\right)-(P-1)\right]\left(A^{\prime}-P B^{\prime}\right)}
$$

i.e. $\frac{A_{1}-p B_{1}}{B_{1}+1} \geq 2 k$

where $k=\frac{n(A-p B)\left(A^{\prime}-p B^{\prime}\right)}{\left(3 B_{1}+p-A_{1}+1\right)\left(3 B^{\prime}+p-A^{\prime}+1\right)+\left[\left(A_{1}-B_{1}\right)-(P-1)\right]\left(A^{\prime}-P B^{\prime}\right)}$

Theroem2.3: If $f(z) \in C(A, B)$ and $g(z) \in C\left(A^{\prime}, B^{\prime}\right)$ then $f(z) * g(z) \in C\left(A_{1}, B_{1}\right)$ where

$$
\begin{aligned}
A_{1} \geq 1, B_{1} \leq & \frac{A_{1}-2 k}{1+2 k} \text { with } \\
& k=\frac{(A-p B)\left(A^{\prime}-p B^{\prime}\right)}{9\left(3 B_{1}+p-A_{1}+1\right)\left(3 B^{\prime}+p-A^{\prime}+1\right)+\left[\left(A_{1}-B_{1}\right)-(P-1)\right]\left(A^{\prime}-P B^{\prime}\right)}
\end{aligned}
$$

Proof : Let us verify with the following example

$$
\begin{aligned}
& f(z)=z^{p}-\frac{(A-p B)}{3(3 B+p-A+2)} z^{P+2} \in C(A, B) \\
& g(z)=z^{p}-\frac{\left(A^{\prime}-p B^{\prime}\right)}{3\left(3 B^{\prime}+p-A^{\prime}+2\right)} z^{p+2} \in C\left(A^{\prime}, B^{\prime}\right)
\end{aligned}
$$

Then

$$
f(z) * g(z)=z^{p}-\frac{(A-p B)\left(A^{\prime}-p B^{\prime}\right)}{9(3 B+p-A+2)\left(3 B^{\prime}+p-A^{\prime}+2\right)} z^{p+1} \in C\left(A_{1}, B_{1}\right)
$$

if

$$
\frac{(p-1)+n\left(B_{1}+1\right)-\left(A_{1}+B_{1}\right)}{A_{1}-p B_{1}}=\frac{9(3 B+p-A+2)\left(3 B^{\prime}+p-A^{\prime}+2\right)}{(A-p B)\left(A^{\prime}-p B^{\prime}\right)}
$$

Simplifying, we get

$$
A_{1}=p(1+2 K)
$$

where $K=\frac{(\mathrm{A}-\mathrm{pB})\left(\mathrm{A}^{\prime}-\mathrm{pB}^{\prime}\right)}{9\left(3 \mathrm{~B}_{1}+\mathrm{p}-\mathrm{A}_{1}+1\right)\left(3 \mathrm{~B}^{\prime}+\mathrm{p}-\mathrm{A}^{\prime}+1\right)+\left[\left(\mathrm{A}_{1}-\mathrm{B}_{1}\right)-(\mathrm{P}-1)\right]\left(\mathrm{A}^{\prime}-\mathrm{PB}^{\prime}\right)}$ 


\section{References}

[1]. A.W.Goodman ,Univalent functions, Vol I, II, Polygonal Publishing House, Washington, N.J.,1983

[2]. H.Silverman, On a close-to-convex function, Proc.Amer.Math.Soc.36, No.2 (1972), 477-484.

[3]. K.S.Padmanabhan and Ganeshan, Convolution of certain class of univalent function with negative coefficient, Indian $J$ Pure Appl.Math.Sept.19 (9) (1998), 880-889.

[4]. S.M.Khairnar and Meena More, Convolution properties of univalent functions with Missing Second Coefficient of Alternating Series,Int.J.Of Math Analysis, Vol.2,2008, No.12,569-580.

[5]. L.V.Ahlfors, Complex Analysis, McGraw Hill Book Co. Inc. New York (1953) 